\title{
Methylmalonyl-CoA Mutase Induction by Cerebral Ischemia and Neurotoxicity of the Mitochondrial Toxin Methylmalonic Acid
}

\author{
Purnima Narasimhan, Robert Sklar, Matthew Murrell, Raymond A. Swanson, and Frank R. Sharp \\ Department of Neurology, University of California, San Francisco, San Francisco, California 94143, and Department of \\ Veterans Affairs Medical Center, San Francisco, California 94121
}

\begin{abstract}
Differential screening of gerbil brain hippocampal cDNA libraries was used to search for genes expressed in ischemic, but not normal, brain. The methylmalonyl-CoA mutase (MCM) cDNA was highly expressed after ischemia and showed a 95\% similarity to mouse and $91 \%$ similarity to the human MCM cDNAs. Transient global ischemia induced a fourfold increase in MCM mRNA on Northern blots from both hippocampus and whole forebrain. MCM protein exhibited a similar induction on Western blots of gerbil cerebral cortex 8 and $24 \mathrm{hr}$ after ischemia. Treatment of primary brain astrocytes with either the branched-chain amino acid (BCAA) isoleucine or the BCAA metabolite, propionate, induced MCM mRNA fourfold. Increased concentrations of BCAAs and odd-chain fatty acids, both of which are metabolized to propionate, may contribute to inducing the MCM gene during ischemia.

Methylmalonic acid, which is formed from the MCM substrate methylmalonyl-CoA and which inhibits succinate dehydrogenase
\end{abstract}

$(\mathrm{SDH})$, produced dose-related cell death when injected into the basal ganglia of adult rat brain. This neurotoxicity is similar to that of structurally related mitochondrial SDH inhibitors, malonate and 3-nitropropionic acid. Methylmalonic acid may contribute to neuronal injury in human conditions in which it accumulates, including MCM mutations and $B_{12}$ deficiency. This study shows that methylmalonyl-CoA mutase is induced by several stresses, including ischemia, and would serve to decrease the accumulation of an endogenous cellular mitochondrial inhibitor and neurotoxin, methylmalonic acid.

Key words: methylmalonic acid; methylmalonyl-CoA mutase; branched-chain amino acids; odd-chain fatty acids; propionate; cerebral ischemia; stroke; excitatory amino acids; vitamin $B_{12}$; astrocytes; 3-nitropropionic acid; succinate dehydrogenase; malonate; hypoxia; mitochondria
Cells can respond to metabolic stress by altering gene expression. The inducible HSP70 heat-shock protein is expressed after a variety of cellular stresses, including heat-shock and ischemia (Brown et al., 1989; Gonzalez et al., 1989; Nowak et al., 1990; Kinouchi et al., 1993; Lindquist, 1994). Low glucose and oxygen and high intracellular calcium induce the glucose-regulated proteins (Lee and Bondy, 1993; Massa et al., 1995, 1996; Vannucci et al., 1996). In the present study, differential screening of brain cDNA libraries was used to identify other genes that are induced during cerebral ischemia. The hippocampus was examined, because this structure is particularly vulnerable to global ischemia (Kirino, 1982; Kirino and Sano, 1984). One of the identified clones coded for the enzyme methylmalonyl-CoA mutase (MCM).

MCM is a mitochondrial enzyme with homodimer subunits between 72,000 and 79,000 Da (Kolhouse et al., 1980; Fenton et al., 1984). Branched-chain amino acids (BCAA) and odd-chain fatty acids are metabolized to propionate, which then is carboxylated to methylmalonyl-CoA (Rosenberg, 1983; Dickson et al., 1994). MCM metabolizes methylmalonyl-CoA to succinyl CoA (Kolhouse et al., 1980; Rosenberg, 1983; Fenton et al., 1984).

Received June 17, 1996; revised Aug. 26, 1996; accepted Aug. 29, 1996.

This work was supported by National Institutes of Health Grants NS28167 and NS14543 (to F.R.S.) and by the Merit Review Program of the Department of Veterans Affairs (to F.R.S. and R.A.S.). We thank Drs. Stephen Sagar and Stephen Massa for their helpful comments and criticism.

Correspondence should be addressed to Dr. Frank Sharp, Department of Neurology (V127), University of California, San Francisco, San Francisco, CA 94143, and Department of Veterans Affairs Medical Center, 4150 Clement Street, San Francisco, CA 94121.

Copyright (C) 1996 Society for Neuroscience $0270-6474 / 96 / 167336-11 \$ 05.00 / 0$
The human and mouse MCM genes have been cloned and sequenced. Mutations of the MCM gene (Ledley et al., 1988, 1990; Ledley, 1990; Wilkemeyer et al., 1990) result in accumulation of methylmalonic acid (Gebarski et al., 1983; Matsui et al., 1983). The severe metabolic and neurological abnormalities and rapid death that occur shortly after birth in patients with no MCM activity indicate that the MCM enzyme is essential for normal brain development (Matsui et al., 1983; Rosenberg, 1983; Ostergaard et al., 1991; Sum et al., 1993). Although the mechanism of the neurotoxicity has not been resolved, recent studies have shown that methylmalonic acid inhibits succinic acid dehydrogenase (SDH) and thereby interferes with Kreb's cycle and mitochondrial electron transport function (Dutra et al., 1991, 1993; Wajner et al., 1992; Toyashima et al., 1995). This led us to postulate that methylmalonic acid might cause neural cell death, because the structurally related SDH inhibitors, malonate and 3-nitropropionic acid, kill neurons in cell culture and in vivo in both experimental animals and humans (Gould and Gustine, 1982; Hamilton and Gould, 1987; Ludolf et al., 1991, 1992; Riepe et al., 1992; Beal et al., 1993a-c, 1994; Greene et al., 1993; Henshaw et al., 1994; Wullner et al., 1994; Behrens et al., 1995; Fu et al., 1995a,b; Greene and Greenamyre, 1995a,b; He et al., 1995; Kirschner et al., 1995; Zeevalk et al., 1995).

The gerbil brain MCM cDNA was sequenced, and Northern and Western blotting were used to confirm that MCM mRNA and protein were induced after brain ischemia. The possible mechanism of MCM induction was explored by determining whether metabolic precursors of methylmalonyl-CoA induce MCM 
mRNA. Finally, the possibility that methylmalonic acid was neurotoxic was examined by injecting it into adult rat basal ganglia. The data suggest that MCM expression may be regulated by BCAA and other sources of propionate. MCM induction would decrease the accumulation of toxic metabolic intermediates, like methylmalonic acid, which would interfere with normal oxidative cellular function and contribute to cell death during periods of stress, including ischemia.

\section{MATERIALS AND METHODS}

Transient forebrain ischemia. Adult male Mongolian gerbils (Tumblebrook Farm, West Brookfield, MA), anesthetized with isoflurane, had both common carotid arteries occluded with microvascular clamps for 10 min (Kirino and Sano, 1984). Rectal temperature was maintained at $37^{\circ} \mathrm{C}$ throughout the procedure. Sham-operated animals had the carotids isolated without carotid occlusion. At various times after ischemia $(4,8,24$ $\mathrm{hr}$ ) animals were deeply anesthetized with isoflurane and decapitated. Brains were removed rapidly. The hippocampi were dissected, frozen on dry ice, and kept at $-85^{\circ} \mathrm{C}$ until processed.

cDNA library construction. Poly $\left(\mathrm{A}^{+}\right)$RNA was isolated from hippocampi $24 \mathrm{hr}$ after ischemia by the Micro-fast track mRNA isolation kit (Invitrogen, San Diego, CA). cDNA was prepared from $2 \mu \mathrm{g}$ of mRNA (BRL cDNA synthesis kit, Bethesda, MD). The cDNA was methylated with EcoRI, blunted with T4 DNA polymerase (Promega, Madison, WI), and ligated to phosphorylated EcoRI linkers (Pharmacia, Piscataway, NJ). The EcoRI digest was size-selected by agarose gel electrophoresis. cDNA ranging in size from 0.3 to $6.0 \mathrm{~kb}$ was selected and ligated into the $\lambda$ ZAPII cloning vector and packaged into phage in vitro with a Gigapack Gold packaging kit (Stratagene, La Jolla, CA). A similar library was constructed from hippocampus of sham-operated "normal" gerbils.

Differential screening of library. The libraries were plated by infecting XL-I blue bacteria at a density of 300 plaques $/ 150 \mathrm{~mm}$ plate. Three plaque lifts onto nitrocellulose filters were done for each plate. Two lifts were probed with ${ }^{32} \mathrm{P}$-labeled ischemic riboprobe and one with ${ }^{32} \mathrm{P}$ labeled riboprobe of sham-operated animals. Plaques that showed high levels of hybridization with the ischemic probe and low levels with the sham probe were isolated for further characterization. Plasmids from the plaques of interest were isolated by using the in vivo excision procedure (Stratagene). The purified plasmids were rescreened by slot-blot hybridization with sham and ischemic radiolabeled cDNA probes. The clones were sequenced with a Sequenase kit (United States Biochemicals, Cleveland, $\mathrm{OH})$.

Northern analysis. The mRNA samples from hippocampus, cortex, stomach, heart, kidneys, and liver of sham and ischemic gerbils were electrophoresed on a formaldehyde-denaturing 1.2\% agarose gel and blotted onto nylon membranes (Maniatis, 1987). The RNA was immobilized on the membrane by UV cross-linking. Then the blots were hybridized in a solution containing $50 \%$ formamide, $5 \times$ SSC, $5 \times$ Denhardt's, $1 \%$ SDS, and $0.5 \mathrm{mg} / \mathrm{ml}$ denatured salmon sperm DNA along with random-primed ${ }^{32} \mathrm{P}$-labeled $500 \mathrm{bp}$ HindIII fragment of MCM cDNA as a probe. The membranes were washed in $2 \times$ SSC and $0.1 \%$ SDS. For the ischemic brain Northern, a 51 base antisense oligodeoxynucleotide to MCM (bases 1360-1471; sequence underlined in Fig. 1) 5'- TGG CAT TCC ACC CAT TTC TTC AAC CTC ATG TAT CAG CTT CAG AGC AGC TTC $-3^{\prime}$ was $3^{\prime}$ end-labeled with ${ }^{32} \mathrm{P}$-dATP (NEN, Boston, MA) and terminal deoxynucleotidyl transferase (BRL) and was purified in an NEN push column. An oligodeoxynucleotide complementary to cyclophilin A (CYC) was used as a control.

Poly $\left(\mathrm{A}^{+}\right)$mRNA samples from different developmental times of rat brains were similarly extracted, electrophoresed, and blotted on nylon membranes. The membranes were hybridized as described above by using the randomly primed ${ }^{32} \mathrm{P}$-labeled MCM cDNA as a probe. The optical densities of the MCM and cyclophilin (CYC) bands on all Northern blots were measured with an MCID computer-based imaging system. The $\mathrm{MCM} / \mathrm{CYC}$ ratios were compared in ischemic versus control brain, in brain at different developmental ages, and for astrocytes treated as described in the next section.

Astrocyte cultures. Astrocytes were prepared according to Hertz (Hertz et al., 1985; Hertz, 1990) with modification (Swanson and Sharp, 1992). One-day-old Sprague Dawley rats (Simonsen, Gilroy, CA) were anesthetized with isoflurane and decapitated. The cortices were dissected free of meninges, minced, and placed in $\mathrm{Ca}^{2+} / \mathrm{Mg}^{2+}$-free HBSS containing 20 units $/ \mathrm{ml}$ papain and $0.5 \mathrm{mg} / \mathrm{ml} \mathrm{DNase}$ I for $30 \mathrm{~min}$. The cells were centrifuged and suspended in Eagle's essential medium containing 10\% fetal bovine serum (HyClone, Ogden, UT) and $2 \mathrm{~mm}$ glutamine. Cell dissociation was completed by trituration through a fire-polished glass pipette. The cells were plated in flasks at a density of $\sim 1 \times 10^{9}$ cells/flask and incubated at $37^{\circ} \mathrm{C}$ in a humidified $5 \% \mathrm{CO}_{2}$ chamber. Cultured cells became confluent at $12-15 \mathrm{~d}$ in vitro, at which time $20 \mu \mathrm{M}$ cytosine arabinoside was added to arrest proliferation of other cell types. The medium was exchanged after $48 \mathrm{hr}$ with fresh medium containing $2.5 \%$ fetal bovine serum and $2 \mathrm{~mm}$ glutamine plus $0.15 \mathrm{~mm}$ dibutyryl cAMP to induce differentiation (Hertz, 1990). The study was repeated on cells from four different batches of astrocyte cultures at 25-34 d in vitro.

Propionate and amino acid treatments. Propionate (5 and $0.5 \mathrm{~mm})$, valine ( 2 and $0.2 \mathrm{~mm}$ ), or isoleucine $(2$ and $0.2 \mathrm{~mm}$ ) (Sigma, St. Louis, MO) were added to three different sets of astrocyte flasks ( $\mathrm{pH} 7.2$, DMEM). Astrocytes in DMEM medium without propionate, isoleucine, or other branched-chain amino acids were used as controls. The astrocytes were harvested $72 \mathrm{hr}$ later, and mRNA was extracted. The RNA was processed and immobilized onto a nylon membrane as described above. The membrane was probed with $500 \mathrm{bp}$ HindIII fragment of MCM cDNA by the hybridization procedure detailed above.

Western analysis. Protein was extracted from control and ischemic cortices and hippocampi of gerbils in Laemmli buffer. The proteins were denatured by boiling. Cellular proteins were separated by $10 \%$ SDSPAGE. The proteins were transferred to a PVDF membrane (Stratagene) by electroblotting at $60 \mathrm{~V}$ for $3 \mathrm{hr}$ and blocked overnight in $0.25 \%$ powdered milk, $0.1 \%$ Tween, and PBS solution. A duplicate membrane was stained with Ponceau red to confirm that an equal amount of protein was loaded in each lane. Immunostaining was performed by using the Vectastain protocol. The blot was incubated for $1 \mathrm{hr}$ with a rabbit anti-human MCM antibody diluted to 1:1000 in blocking buffer. Then it was rinsed in wash buffer $(0.1 \%$ Tween/PBS) and incubated for $1 \mathrm{hr}$ in biotinylated secondary antibody (anti-rabbit) diluted 1:2000 in blocking buffer. The blot was rinsed in wash buffer and treated with avidin-HRP solution for $30 \mathrm{~min}$. After rinsing with wash buffer, MCM protein was visualized by using $0.1 \%$ diethylaminobenzidine in $0.1 \mathrm{M}$ Tris, $\mathrm{pH} 7.2$, and $0.005 \% \mathrm{H}_{2} \mathrm{O}_{2}$.

Intracerebral injections of methylmalonic acid. Adult Sprague Dawley rats $(250 \mathrm{gm})$ were anesthetized with isoflurane. Burr holes were drilled in the skull bilaterally, $2.6 \mathrm{~mm}$ lateral to bregma. A 30 gauge needle was advanced $5.0 \mathrm{~mm}$ into the striatum. Two microliters of a given solution were infused into the striatum over $5 \mathrm{~min}$. The solutions injected included $0.9 \%$ saline $(n=4) ; 100 \mathrm{mg} / \mathrm{ml}$ of citric acid buffered to $\mathrm{pH} 7.1$ with $0.1 \mathrm{M} \mathrm{pH} 7.1$ phosphate buffer $(\mathrm{PB})$ and $10 \mathrm{~N} \mathrm{NaOH}(n=$ 4); and methylmalonic acid, in concentrations of 1, 10, 25, 75, 100, 500, and $600 \mathrm{mg} / \mathrm{ml}$ ( $n=6$ for each dose). Methylmalonic acid was dissolved in $\mathrm{PB}, \mathrm{pH} 7.1$, and the $\mathrm{pH}$ was adjusted to 7.1 with $10 \mathrm{~N} \mathrm{NaOH}$. Sodium azide $(100 \mathrm{mg} / \mathrm{ml}, \mathrm{pH}$-adjusted to 7.1), an inhibitor of the cytochrome oxidase/Complex IV in mitochondria, was also infused into striatum as a "positive" control $(n=4)$. Animals were anesthetized $3 \mathrm{~d}$ later and perfused, and the brains were removed and sectioned on a vibratome. Sections were mounted on slides and Nissl-stained. Lesion areas at the level of the anterior commissure were quantified for each subject with an MCID computer-based image analysis system to measure the lesion area on the basis of a density threshold. Optical density measurements of the average Nissl optical density of the contralateral intact striatum were performed, and a value $15 \%$ less than this was used to set a threshold below which a lesion was defined in the injected striatum. Then the computer was used to detect and compute automatically the areas on each section showing decreased optical densities. Microscopic examination of these lesion areas showed loss of most neurons and some glia. The area of the lesion was measured on six sections at the level of the anterior commissure from each subject and averaged. Differences in these averages between groups were analyzed with ANOVA, followed by a Scheffe analysis for comparisons between groups.

\section{RESULTS}

\section{Isolation of methylmalonyl-CoA mutase (MCM) CDNA}

One of the clones obtained from the differential screening of the ischemic and sham cDNA libraries coded for the enzyme methylmalonyl-CoA mutase. The MCM clone was digested with EcoRI and subcloned in pBluescript (Stratagene), resulting in pM7 and pML. These clones, $800 \mathrm{bp}$ and $2 \mathrm{~kb}$ in length, 
$5^{\prime}$

acg aat ggg tca gtt ctc att tcc ttt tgg gag tat cca tgc ttc acc atg ttg aga gct aag aat cag ctt ttt ttg tta 8 $\begin{array}{llllllllllllllllllllll}-48 & +1 & M & L & R & A & K & N & Q & L & F & L & L\end{array}$ tca ccc cac tac ctg aag cag cta aaa gat cca tca tcc tcc acg tgg aaa cgg ctc ctg cat cag cag aaa acc ctt cac 16 $\begin{array}{llllllllllllllllllllllllllll}S & P & H & Y & \text { L } & K & Q & \text { L } & K & \text { K } & \text { P } & \text { S } & \text { S } & \text { S } & \text { T } & \text { W } & \text { K } & \text { R } & \text { L } & \text { L } & H & Q & Q & K & T & L & H\end{array}$ cca gaa tgg gct gca ctg gcc aaa aag cag ctg aaa ggc aaa aac cca gag gac ctc ata tgg cgt acc cca gaa ggg atc 24 $\begin{array}{lllllllllllllllllllllllllll}P & E & W & A & A & \text { L } & A & K & K & Q & I & K & G & K & N & P & E & D & I & I & W & R & T & P & E & G & I\end{array}$ tct ata aag ccc tta tat tcc agg gca gac aca atg gac tta cet gaa gaa ctt cca gga gtg aag tta ttc aca cga gga 32 $\begin{array}{lllllllllllllllllllllllllll}S & I & K & P & I & Y & S & R & A & D & T & M & D & L & P & E & E & L & P & G & V & K & L & F & T & R & G\end{array}$ ccg tat $\operatorname{ccc}$ acc atg tat acc tat agg cca tgg acc atc cgt cag tat gca ggc ttt agt act gtg gaa gaa agc tat aaa 40

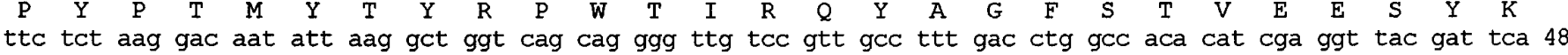
$\begin{array}{llllllllllllllllllllllllllll}\mathrm{F} & \mathrm{S} & \mathrm{K} & \mathrm{D} & \mathrm{N} & \mathrm{I} & \mathrm{K} & \mathrm{A} & \mathrm{G} & \mathrm{Q} & \mathrm{Q} & \mathrm{G} & \mathrm{L} & \mathrm{S} & \mathrm{V} & \mathrm{A} & \mathrm{F} & \mathrm{D} & \mathrm{L} & \mathrm{A} & \mathrm{T} & \mathrm{H} & \mathrm{R} & \mathrm{G} & \mathrm{Y} & \mathrm{D} & \mathrm{S}\end{array}$ gac aac cct cga gtt cgt gga gat gtt gga atg gcg gga gtt gct att gac act gta gaa gac acc aaa atc cta ttt gat 56 $\begin{array}{lllllllllllllllllllllllllll}D & N & P & R & V & R & G & D & V & G & M & A & G & V & A & I & D & T & V & E & D & T & K & I & L & F & D\end{array}$ gga att cet tta gaa aaa atg tcc gtt tcc atg acc atg aat gga gca gtt atc cca atc ctt gcg acg ttt ata gta acc 64 $\begin{array}{lllllllllllllllllllllllllll}G & I & P & L & E & K & M & S & V & S & M & T & M & N & G & A & V & I & P & I & L & A & T & F & I & V & T\end{array}$ ggg gaa gaa caa ggt gtg $\mathrm{Ccg}$ aaa gag aag ctc act ggt aca att cag aat gat atc cta aag gag ttt atg gtc aga aat 72 $\begin{array}{lllllllllllllllllllllllllll}G & E & E & Q & G & V & P & K & E & K & L & T & G & T & I & Q & N & D & I & I & K & E & F & M & V & R & N\end{array}$ aca tat atc ttt cCc cca gaa cca tcc atg aaa att att get gac att ttc caa tac aca gca cag cac atg cca aaa ttt 81 $\begin{array}{llllllllllllllllllllllllllll}T & Y & I & F & P & P & E & P & S & M & K & I & I & A & D & I & F & Q & Y & T & A & Q & H & M & P & K & F\end{array}$ aat cca ttt tcg att agt ggg tac cat atg cag gga gcg gga gct gat gcc att cta gaa ctg gcc tat acc atc gcg gat 89 $\begin{array}{lllllllllllllllllllllllllll}N & P & F & S & I & S & G & Y & H & M & Q & G & A & G & A & D & A & I & \text { L } & E & \text { L } & \text { A } & Y & \text { T } & \text { I } & \text { A } & \text { D }\end{array}$ ggc ttg gag tac tgc aga acc gga ctc cag get ggt ctc aca att gat gaa ttt gca cca agg ttg tct ttc ttc ttg gga 97 $\begin{array}{lllllllllllllllllllllllllll}G & L & E & Y & C & R & T & G & I & Q & A & G & I & T & I & D & E & F & A & P & R & L & S & F & F & L & G\end{array}$ att ggg atg aat ttc tac atg gaa ata gca aag atc cga get ggg agg aga ctc tgg gct cac tta ata gaa aaa atg ttc105

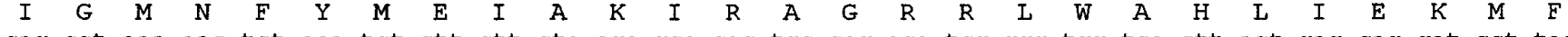
cag cct aaa aac tct aaa tct ctt ctt cta aga gca cac tgc cag aca tcg ggg tgg tca ctt act gag cag gat cct tacl13

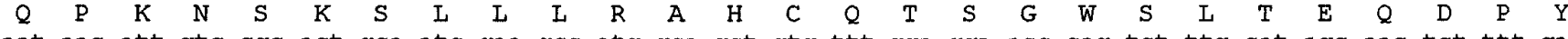
at aac att gtc cgc act gca atc gaa gcc atg gca gct gtg ttt gga gga acc cag tct ttg cat agc aac tct ttt gat121 $\begin{array}{lllllllllllllllllllllllllll}N & N & I & V & R & T & A & I & E & A & M & A & A & V & F & G & G & T & Q & S & I & H & S & N & S & F & D\end{array}$ gaa gct ctg gge ttg cca act gtg aaa agc gct cga att gcc cga aat aca caa atc atc att caa gaa gaa tct ggg att129 $\begin{array}{llllllllllllllllllllllllllll}E & A & \text { L } & G & \text { L } & \text { P } & \text { T } & \text { V } & \text { K } & \text { S } & \text { A } & \text { R } & \text { I } & \text { A } & \text { R } & \text { N } & \text { T } & Q & \text { I } & \text { I } & \text { I } & Q & E & E & S & G & I\end{array}$ CCC aaa gtg gec gat cct tgg gga gga tca tac atg atg gaa tct ctc aca aat gat gtg ttt gaa get gct ctg aag ctg137 $\begin{array}{lllllllllllllllllllllllllll}P & K & V & A & D & P & W & G & G & S & Y & M & M & E & S & L & T & N & D & V & F & E & A & A & L & K & I\end{array}$ ata cat gag gtt gaa gaa atg got gga atg gcc aaa get gta gct gaa gga ata cct aa ctt cga att gaa gaa tgt get145

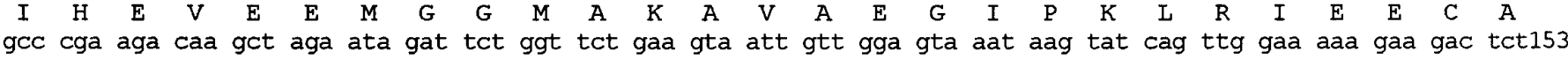
$\begin{array}{llllllllllllllllllllllllllll}A & R & R & Q & A & R & I & D & S & G & S & E & V & I & V & G & V & N & K & Y & Q & L & E & K & E & D & S\end{array}$ gtg gaa gtc ctg gcc att gac aac act tca ggt cgc aag aag cag att gag aaa atc aag aag atc aaa tcc agc cgg gat162 $\begin{array}{ccccccccccccccccccccccccccc}V & E & V & L & A & I & D & N & T & S & G & R & K & K & Q & I & E & K & I & K & K & I & K & S & S & R & D \\ \text { caa } & \text { gct } & \text { ttg } & \text { gct } & \text { gaa } & \text { cgg } & \text { gcc } & \text { ctc } & \text { aga } & \text { cgg } & \text { ctt } & \text { acc } & \text { cag } & \text { tgt } & \text { gct } & \text { gcc } & \text { agt } & \text { gga } & \text { gat } & \text { cgc } & \text { aat } & \text { att } & \text { ttg gct } & \text { ctt } & \text { gca } & \text { gtgl } & \text { gto }\end{array}$


gat cga gct cgt gca aga tgt cga gtt gga gaa atc aca gat gct ttg aaa aag ata ttt ggt aag cat aaa gct aat gat178 $\begin{array}{llllllllllllllllllllllllllll}D & R & A & R & A & R & C & R & V & G & E & I & T & D & A & I & K & K & I & F & G & K & H & K & A & N & D\end{array}$ cgc atg gtg agt gga gca tat cgg cag gag ttt gga gaa agt aaa gag att aca tct gcc atc aag aga gtt gat aag ttcl86 $\begin{array}{llllllllllllllllllllllllllll}R & M & V & S & G & A & Y & R & Q & E & F & G & E & S & K & E & I & T & S & A & I & K & R & V & D & K & F\end{array}$ aag gaa cgt gaa ggt cgc cga cct cgt ctt ctt gtg gca aaa atg ggg caa gat ggc cat gac aga gga gcg aag gtc att194 $\begin{array}{lllllllllllllllllllllllllll}K & \text { E } & \text { R } & \text { E } & G & \text { R } & \text { R } & \text { P } & \text { R } & \text { L } & \text { L } & \text { V } & \text { A } & \text { K } & \text { M } & \text { G } & \text { Q } & \text { D } & \text { G } & \text { H } & \text { D } & \text { R } & \text { G } & \text { A } & K & \text { V } & I\end{array}$ gct aca gga ttt gct gat ctt ggt ttt gat gtg gaa ata ggg cet ctt ttt cag act ccc cgt gaa gtg gca cac gag gct202 $\begin{array}{lllllllllllllllllllllllllllllllll}A & T & G & F & A & D & L & G & F & D & V & E & I & G & P & L & F & Q & T & P & R & E & V & A & H & E & A\end{array}$ gtg gac gca gat gtg cat gct gtg ggt gtc agc aca ctt gcc get ggt cac aaa acc ctc gtt cct gag ctt atc aaa gaa210 $\begin{array}{lllllllllllllllllllllllllllll}V & D & A & D & V & H & A & V & G & V & S & T & L & A & A & G & H & K & T & L & V & P & E & I & I & K & E\end{array}$ ctg tct gcc ctg ggt cgg cca gat atc ctt gtc atg tgt ggg gge gtg att cca cca cag gat tat gac ttt ctg tat gag218 $\begin{array}{lllllllllllllllllllllllllllll}I & S & A & \text { L } & G & R & P & D & I & I & V & M & C & G & G & V & I & P & P & Q & D & Y & D & F & L & Y & E\end{array}$ ttt ggt gtt tcc aag gta ttt ggt cet gga acc cgg att ccc aag gcc gcc gtt cag gtg ctt gag gac att gag aag tgt226 $\begin{array}{llllllllllllllllllllllllllll} & F & G & V & S & K & V & F & G & P & G & T & R & I & P & K & A & A & V & Q & V & L & E & D & I & E & K & C\end{array}$ ttg gca gag aag cag caa tct gcg taa cac ctg gat ctt tag tta tac tcg aag aga gta ata aaa cta tgt ctt ctg gta234 I $\quad A \quad E \quad K \quad Q \quad Q \quad S \quad A$

att caa aca cet gtt aat gtg ttt get ttg gaa gcc ttt cat taa aag gca gtg att ata aat gtg cag tca tgc taa tac243 aca tat tta gag agc ctg agt taa aat atg aaa atc tga aaa tca tct aca cca agt att tcc aaa gta cat tat ttc aga251 aaa ata aaa tcc ggc gct act gct tag aga taa taa tac aat tat tta taa taa ctt gtg ttt tcg ttt tcg cgt tcg ttt259 gtt tga gaa cag g 2605

$$
3^{\prime}
$$

Figure 1. Primary sequence of gerbil methylmalonyl-CoA mutase, including amino acid sequence. The 51 bp oligonucleotide sequence used for some of the Northern blots is underlined.

were used to sequence the gene. The 2608 bp nucleotide coding sequence of the gerbil MCM cDNA, along with the 5' and $3^{\prime}$ untranslated regions, is shown in Figure 1. The deduced amino acid sequence of the protein was 749 amino acids. The gerbil amino acid sequence exhibited $94 \%$ similarity to the mouse and $91 \%$ to the human MCM proteins (Fig. 2). There was also a 48 


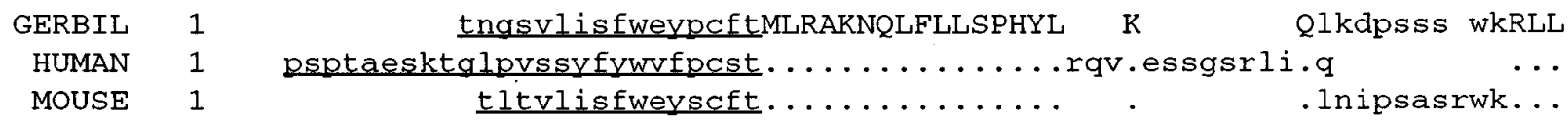

GERBIL 47 HQQkLLHPEWAaLAKKQLKGKNPEDLIWrTPEGISIKPLYSRADTmDLPEELPGVK1FTRGPYPTMYTYR

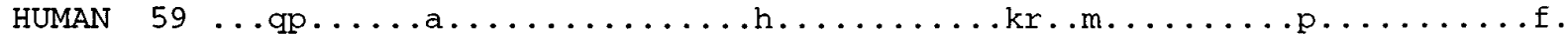

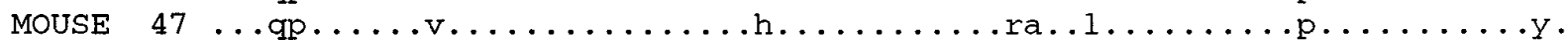

GERBIL 117 PWTIRQYAGFSTVEESNKFSKDNIKAGQQGLSVAFDLATHRGYDSDNPRVRGDVGMAGVAIDTVEDTKIL

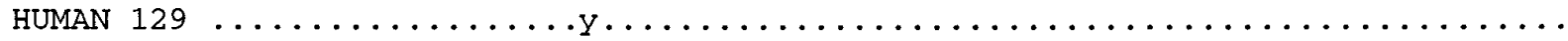

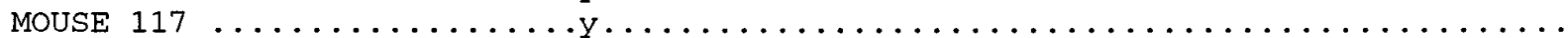

GERBIL 187 FDGIPLEKMSVSMTMNGAVIPILATFIVTGEEQGVPKEKLTGTIQNDILKEFMVRNTYIFPPEPSMKIIA

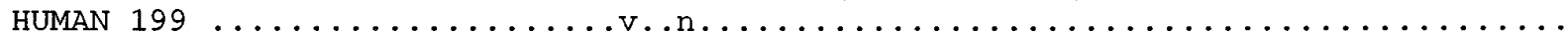

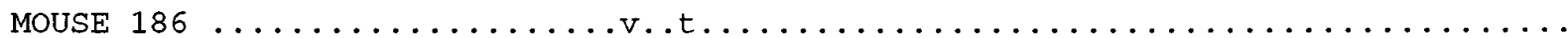

GERBIL 257 DIFqYTAqHMPKFNpfSISGYHMQgAGaDAILELAYTIADGLEYCRTGLQAGLTIDEFAPRLSFFIGIGM



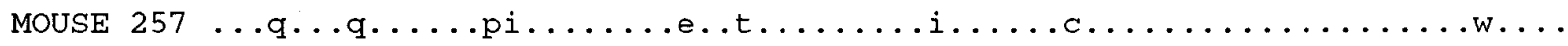

GERBIL 327 NFYMEIAKIRAGRRLWAHLIEKMFQPKNSKSLLLRAHCQTSGWSLTEQDPYNNIVRTAIEAMAAVFGGTQ HUMAN 339

MOUSE 327

.......m.

GERBIL 397 SLHSNSFDEALGLPTVKSARIARNTGIIIQEESGIPKVADPWGGSYMMESLTNDVFEAALKLIhEVEEMG

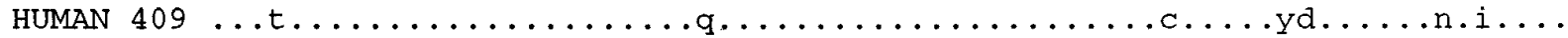

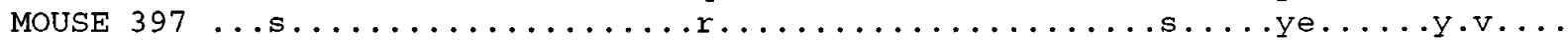

GERBIL 467 GMAKAVAEGIPKLRIEECAARRQARIDSGSEVIVGVNKYqLEKEDSVeVLAIDntSgRkkQIEKiKKIKS

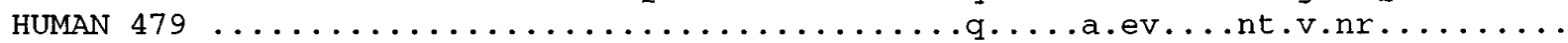

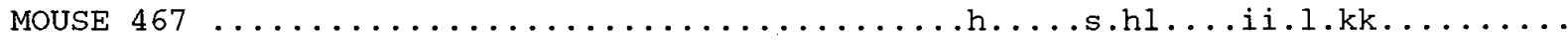

GERBIL 537 SRDQALAErALrrITqCAASGDrNILALAVDraRARCrVgEITDAIKKIFGKHKANDRMVSGAYRQEFGE

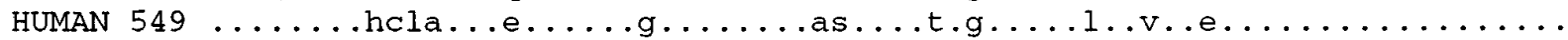

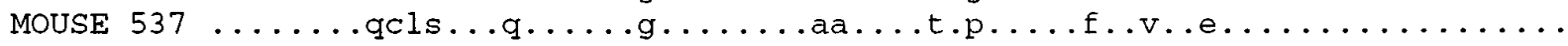

GERBIL 607 SKEITSAIKRVAKFkEREGRRprLLVAKMGqDGHDRGAKVIATGFADLGFDVEIGPLFQTPREVAheAVD

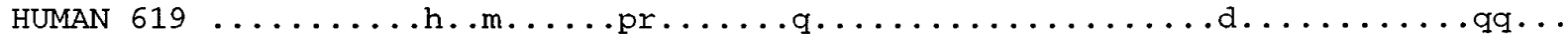

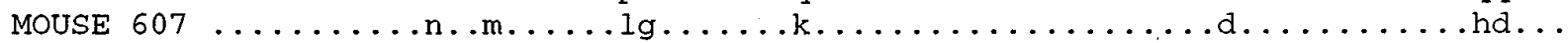

GERBIL 677 ADVHAVGVST1AAGHKTLVPELIKELSALGRPDILVMCGGVIPPQDYAFLYEEGVSkVFGPGTRIPkAAV

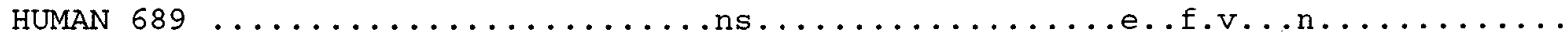

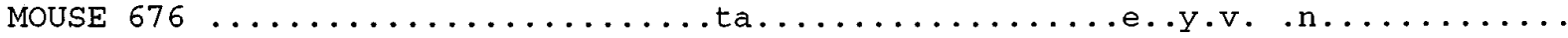

GERBIL 748 QVLEDIEKCLaeKQQSa*

HUMAN $759 \ldots$......ek.... v $^{\star}$

MOUSE 746 .d............ $v^{*}$

Figure 2. Comparison of gerbil, mouse, and human amino acid sequences for methylmalonyl-CoA mutase. Dots indicate identical amino acids across species. The mitochondrial leader sequence is underlined. 


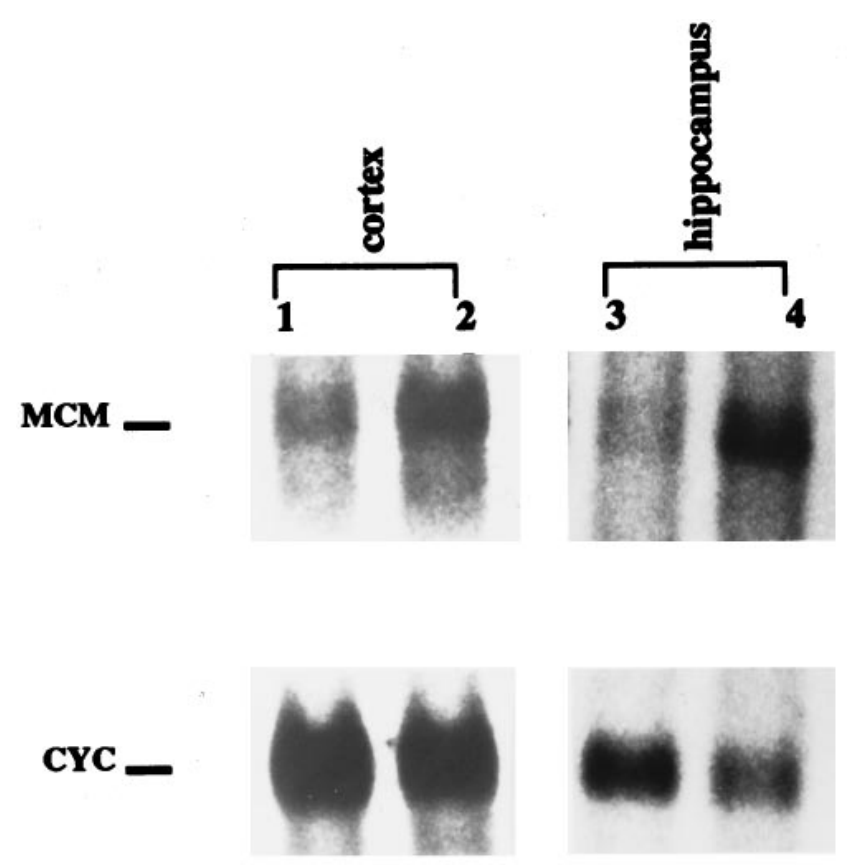

Figure 3. Northern blots of methylmalonyl-CoA mutase (MCM) mRNA from cortex (lanes 1,2) and hippocampus (lanes 3, 4) and from control sham-operated gerbils (lanes 1,3) and $8 \mathrm{hr}$ after $10 \mathrm{~min}$ of global ischemia (lanes 2, 4). The cyclophilin $(C Y C)$ bands show that fairly equal amounts of mRNA were loaded in each lane. This is representative of the three separate blots on six separate subjects that were performed.

bp mitochondrial targeting peptide sequence at the $5^{\prime}$ region that was similar to the mouse and human MCM gene.

\section{MCM mRNA in ischemic brain}

Northern analysis of ischemic and control tissues was performed. Poly $\left(\mathrm{A}^{+}\right)$mRNA was isolated from cortex and hippocampus from normal gerbils and from gerbils $24 \mathrm{hr}$ after $10 \mathrm{~min}$ of global ischemia. The Northern blots, probed by using the 51 mer oligonucleotide for MCM, showed induction of MCM mRNA in both cortex and hippocampus of ischemic, as compared with sham control, animals (Fig. 3). The $2.9 \mathrm{~kb}$ MCM transcript was increased approximately three- to fourfold in both cortex (3.4-fold; $n=4)$ and hippocampus (4.1-fold; $n=4)$, using the cyclophilin mRNA as a control.

\section{MCM protein expression in ischemic brain}

Induction of a gene characterized by an increase in its mRNA is not always accompanied by an elevation of protein expression after cerebral ischemia (Nowak et al., 1990; Kinouchi et al., 1993). To determine whether the protein was also induced, we analyzed the gerbil cortex. There was marked induction of the MCM protein on Western blots of cortex at $8 \mathrm{hr}$ and $24 \mathrm{hr}$ after $10 \mathrm{~min}$ of global ischemia (Fig. 4), compared with little protein being present in the control cortex (Fig. 4, lane 1). The same pattern was observed on three separate Western blots from three separate sets of ischemic and control brains. The polyclonal rabbit anti-human MCM antibody used recognizes MCM and other proteins, including the 66-69 kDa albumin band (Fig. 4) just below MCM, as previously reported by Ledley et al. (1988). The protein loading in the three different lanes was judged to be similar because of equal staining of the albumin bands (Fig. 4) and the equal staining of the

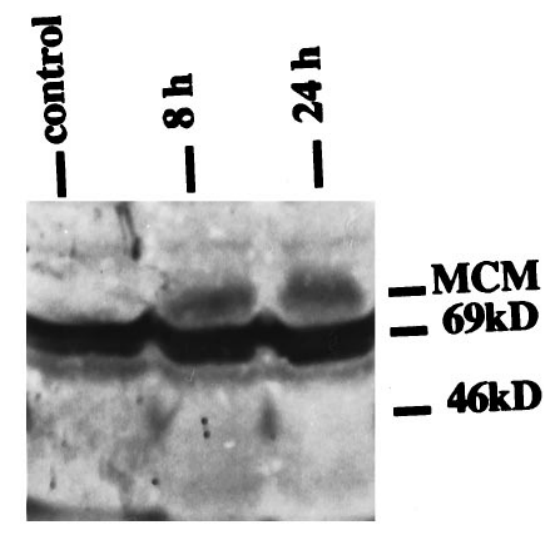

Figure 4. Western blot using a polyclonal antibody to methylmalonylCoA mutase $(M C M)$ showing little MCM protein in control gerbil brain and marked induction of MCM protein in the gerbil brain 8 and $24 \mathrm{hr}$ after 10 min of global ischemia. The polyclonal antibody stained other bands, including albumin at $\sim 69 \mathrm{kDa}$, which shows that similar amounts of protein were loaded in each lane.
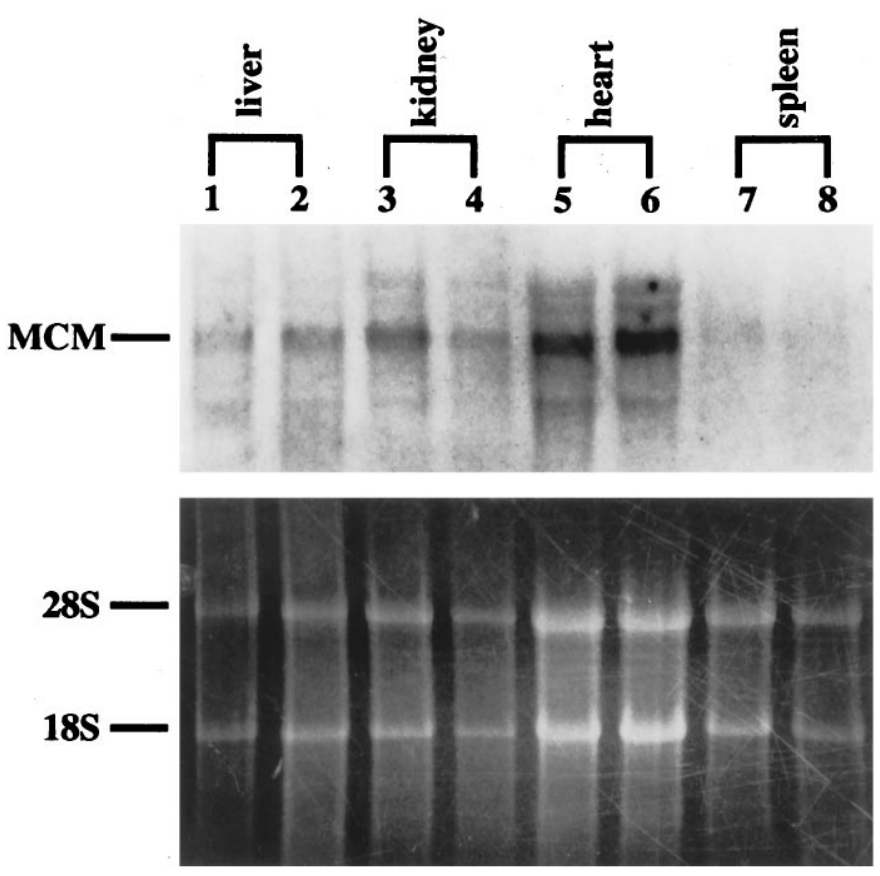

Figure 5. Northern blots of methylmalonyl-CoA mutase (MCM) mRNA from different organs, including the liver (lanes 1,2), kidney (lanes 3, 4), heart (lanes 5,6) and spleen (lanes 7,8). The ethidium bromide-stained gel (bottom) showed that more total RNA had been loaded in the heart lanes (lanes 5, 6). $18 S$ and $28 S$ indicate ribosomal RNA bands. Lanes 1, 3, 5 , and 7 are from the same animal; lanes 2, 4, 6, and 8 are from another animal.

lanes on the duplicate Ponceau red-stained blots (data not shown).

\section{Tissue distribution of MCM mRNA}

Total RNA isolated from liver, kidney, heart, spleen, and lungs from sham controls and cerebral ischemic animals was probed with a $500 \mathrm{bp}$ HindIII fragment from the coding region of the MCM cDNA. MCM mRNA was expressed in most organs (Fig. 5) except the lung (data not shown). A similar tissue distribution has been reported in normal mouse tissues by Wilkemeyer et al. (1993). There were no significant differences in MCM expression 

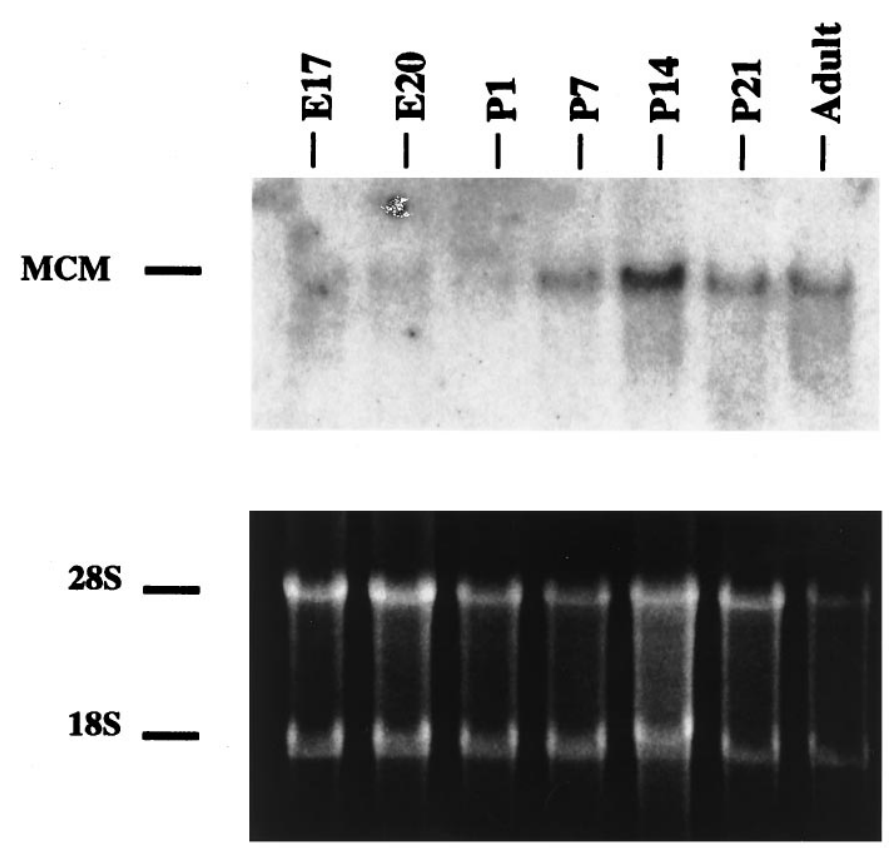

Figure 6. Northern blots of methylmalonyl-CoA mutase (MCM) mRNA from whole brains of animals at different ages: embryonic days 17 (E17) and 20 (E20); postnatal days $P 1, P 7, P 14$, and $P 21$; and from the adult brain (Adult). The ethidium bromide-stained gel (bottom) showed that the lanes were loaded fairly evenly with mRNA.

levels in the sham, as compared with ischemic animals, in any organ other than the brain (data not shown).

\section{MCM gene expression in brain during development}

MCM gene expression was also examined in the developing brain (Dutra et al., 1991; Wajner et al., 1992). The HindIII 500 bp fragment was used to probe poly $\left(\mathrm{A}^{+}\right)$mRNA of embryonic (E17, E19), postnatal (P1, P7, P14, P21), and adult rat brains. The MCM mRNA was not detectable in embryonic brain, was slightly induced by $\mathrm{P} 1$, and peaked at $14 \mathrm{~d}$ after birth (Fig. 6). MCM mRNA decreased at P21 and declined to low adult levels thereafter (Fig. 6).

\section{Induction of MCM with propionate and isoleucine}

Propionyl CoA, which is the source of methylmalonyl-CoA, is derived from the metabolism of BCAA and odd-chain fatty acids (see Fig. 9). Because we postulated that the induction of MCM during ischemia and during development was related to increases in these metabolic precursors, cultured astrocytes were treated with either $5 \mathrm{~mm}$ propionate or $2 \mathrm{~mm}$ isoleucine. Both propionate and isoleucine markedly induced MCM mRNA in treated astrocytes, as compared with control astrocytes incubated in standard MEM without isoleucine or propionate (Fig. 7). The same results were obtained in three separate experiments using three separate batches of cells and three separate runs of the Northern blots. Valine, another BCAA, also induced MCM mRNA (data not shown). Lower concentrations of propionate $(0.5 \mathrm{mM})$ and isoleucine $(0.2 \mathrm{~mm})$ also induced MCM mRNA, but to a lesser degree (data not shown). No experiments were conducted to determine whether BCAA would also induce the enzymes that metabolize BCAA to glutamate (see Fig. 9). The finding that MCM mRNA can be induced in cultured astrocytes suggests that it might also be localized to astrocytes in vivo. Further studies, however, will be

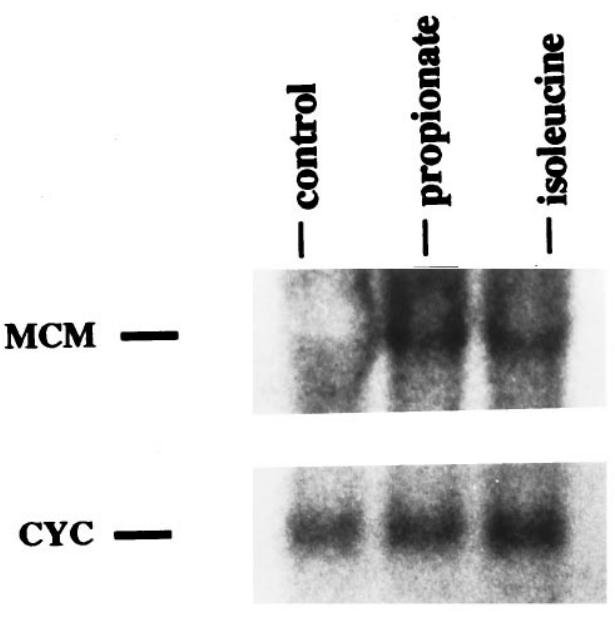

Figure 7. Northern blots of methylmalonyl-CoA mutase (MCM) mRNA from cultured astrocytes exposed for $72 \mathrm{hr}$ to $5 \mathrm{~mm}$ propionate (propionate) or to $2 \mathrm{~mm}$ isoleucine (isoleucine), as compared with control astrocytes (control). The cyclophilin bands $(C Y C)$ show the loading of RNA in each lane.

required to determine which cells express MCM mRNA and protein after ischemia in vivo.

\section{Neurotoxicity of methylmalonic acid injections}

The possible neurotoxicity of methylmalonic acid was examined because it, like malonate and 3-nitropropionic acid, inhibits succinate dehydrogenase (Dutra et al., 1993). The $(2 \mu \mathrm{l})$ injections of saline (Fig. $8 A, C, E$ ) and citric acid (Fig. $8 E$ ) into the striatum did not produce any discernible lesion in any of the animals. Injections $(2 \mu \mathrm{l})$ of the lower doses of methylmalonic acid, including 10, 25, 50 (Fig. $8 E$ ), and $75 \mathrm{mg} / \mathrm{ml}$, did not produce striatal lesions ( $n=6$ at each dose; data not shown). The $100 \mathrm{mg} / \mathrm{ml}$ dose of methylmalonic acid $(n=6)$ produced moderate-sized lesions of striatum (Fig. $8 B, D, E)$, and the highest doses $(n=8)$ of methylmalonic acid $(600 \mathrm{mg} / \mathrm{ml})$ produced very large striatal lesions (Fig. $8 E)$. Injections of sodium azide $(100 \mathrm{mg} / \mathrm{ml})$ also produced striatal lesions (Fig. $8 E$ ). The lower $(100 \mathrm{mg} / \mathrm{ml})$ doses of methylmalonic acid produced selective neuronal loss with preservation of some glial cells within the lesions (Fig. 8D).

\section{DISCUSSION}

These findings demonstrate that methylmalonyl-CoA mutase (MCM) is induced in brain shortly after birth and that MCM can be induced after cerebral ischemia. It is hypothesized that accumulation of branched-chain amino acids and odd-chain fatty acids in brain during development or ischemia increases propionate levels, which in turn induces MCM. Induction of MCM would decrease the accumulation of methylmalonic acid, which has been shown to inhibit succinate dehydrogenase and is shown in this study to injure brain. Methylmalonic acid could contribute to cellular injury by lowering cellular $\mathrm{pH}$, by inhibiting succinate dehydrogenase $(\mathrm{SDH})$ and decreasing the production of GABA from Kreb's cycle intermediates, and by inhibiting SDH and decreasing the generation of ATP via the mitochondrial electron transport chain. Because methylmalonic acid is known to accumulate in brain in several human pathological conditions, including MCM mutations and $\mathrm{B}_{12}$ deficiency, methylmalonic acid may contribute to the cellular injury in the nervous system observed in these conditions.

MCM was cloned first from the liver and has been studied 
primarily in liver (Ledley et al., 1988, 1990; Ledley, 1990; Wilkemeyer et al., 1990, 1993). MCM function in brain has received less attention (Mitzen and Koeppen, 1984; Dutra et al., 1991, 1993; Riley et al., 1991; Dickson et al., 1994; Toyashima et al., 1995) despite the brain abnormalities that occur with MCM mutations (Gebarski et al., 1983; Matsui et al., 1983; Roodhooft et al., 1990; Gerhardt et al., 1991; Ostergaard et al., 1991; Crane et al., 1992; Stockler et al., 1992; Sum et al., 1993; Brismar and Ozand, 1994; He et al., 1995). The complete coding region from gerbil brain MCM cDNA was sequenced, along with $150 \mathrm{bp}$ of $5^{\prime}$ and $254 \mathrm{bp}$ of $3^{\prime}$ untranslated regions. Although there is no poly $\left(\mathrm{A}^{+}\right)$tail, a possible adenylation sequence of AAATAAA is seen at nucleotide position 2572 . The cDNA and the amino acid sequence show high similarity to both the mouse and human liver genes (Ledley et al., 1988; Wilkemeyer et al., 1990). There do not seem to be any large differences in the coding of the liver and brain MCM genes, although MCM in brain exists only as the active holoenzyme, whereas a significant fraction of MCM activity in liver and other tissues exists as inactive apoenzyme (Wilkemeyer et al., 1993). This might relate to transcriptional, translational, or post-translational differences of the gene in different organs.

This study demonstrates the inducibility of the MCM gene in the brain and suggests that MCM could function as a stress gene to abrogate accumulation of methylmalonyl-CoA and its neurotoxic metabolite, methylmalonic acid. Because some Kreb's cycle enzymes are induced by their substrates, we postulated that MCM induction might be attributable to metabolic precursors of succinate. This idea was supported partly by the finding that the BCAA isoleucine and its metabolite, propionate, induced MCM in cultured astrocytes. It is not known whether the concentrations of BCAAs or propionate would reach concentrations sufficient to induce MCM via this mechanism in vivo. The molecular mechanism by which this would occur is also unknown. Although propionate induces MCM mRNA, this does not prove whether propionate itself acts on the MCM gene. The murine MCM promoter contains AP2 and CRE elements that may account for different tissue levels of MCM mRNA (Wilkemeyer et al., 1993). Although the present study provides further evidence for MCM regulation, it is uncertain whether propionate, another MCM substrate, or an unrelated pathway would activate adenylate cyclase or protein kinases to induce MCM via the CRE and/or AP2 elements in the MCM promoter.

The extracellular concentrations of many amino acids increase during cerebral ischemia (Erecinska et al., 1984). Although extracellular concentrations of excitatory amino acids increase the most, BCAA also increase (Erecinska et al., 1984; Drejer et al., 1985; Barks and Silverstein, 1992). If intracellular concentrations of BCAA also increased during ischemia, this could contribute to MCM induction in ischemic brain, because propionate is produced during the degradation of the branched-chain amino acids isoleucine, valine, methionine, and threonine (Matsui et al., 1983; Rosenberg, 1983; Harper et al., 1984) (Fig. 9).

Because propionate induces MCM in cultured astrocytes, sources of propionate other than BCAA also should induce MCM. Figure 9 shows that odd-chain fatty acids are also important sources of propionate (Kishimoto et al., 1973; Rosenberg, 1983). Cerebral ischemia initiates lipid peroxidation, which results in the breakdown of cellular phospholipids and the release of both even- and odd-chain free fatty acids (Bazan, 1970; Yoshida et al., 1986; Nakano et al., 1990). Although most fatty acids have an even number of carbon atoms and are metabolized via $\beta$-oxidation, odd-chain fatty acids represent a small percentage of fatty acids in normal brain. This percentage increases in pathological states and during development (Kishimoto et al., 1973; Rosenberg, 1983; Wendel et al., 1993; Sbai et al., 1994). Odd-chain fatty acids are metabolized via $\alpha$-oxidation to acetyl CoA and propionyl CoA. MCM is essential for breakdown of these odd-chain fatty acids (Fig. 9). The induction of MCM by lipid peroxidation in ischemic brain could be attributable, at least in part, to increased production of odd-chain fatty acids that would be metabolized to propionic acid. The peak expression of MCM mRNA in rat brain for the weeks after birth also could be attributable to high levels of odd-chain fatty acids during the period of myelinogenesis.

The brain abnormalities seen in patients with MCM mutations suggest that methylmalonic acid or other succinate precursors damage the brain. Methylmalonic acid could contribute directly to tissue acidosis by inhibiting the Kreb's cycle and promoting glycolytic metabolism of glucose to lactate. Methylmalonic acid may also injure the brain by inhibiting pyruvate carboxylase; the transmitochondrial shuttle of malate; glycine uptake into synaptosomes; the intramitochondrial glycine cleavage enzyme, carbamyl phosphate synthetase I; and $N$-acetylglutamate synthetase (Halperin et al., 1971; Lopex-Lahoya et al., 1981; Rosenberg, 1983; Wajner et al., 1992; Toyashima et al., 1995).

Methylmalonic acid also inhibits succinate dehydrogenase in brain slices, resulting in increased glucose uptake, increased lactate, and decreased ATP (Dutra et al., 1991; 1993; Wajner et al., 1992; Toyashima et al., 1995). This inhibition of SDH by methylmalonic acid occurs with a $K_{\mathrm{i}}$ of $2.3 \mathrm{~mm}$, a concentration achieved in brain and serum of patients with MCM mutations (Dutra et al., 1993). If methylmalonic acid also accumulates during cerebral ischemia, this would contribute to inhibition of the SDH containing Complex II of the mitochondrial electron transport chain observed when cerebral blood flow falls to $20 \mathrm{cc} / 100 \mathrm{gm}$ per minute (Allen et al., 1995). It is not known whether the methylmalonic acid levels achieved in cerebral ischemia reach $2.3 \mathrm{mM}$, which would inhibit SDH enzyme activity or the higher levels that would kill neurons directly (Fig. $8 E$ ).

Inhibition of SDH by methylmalonic acid may be the primary mechanism by which methylmalonic acid damages the brain. The structurally related molecules malonate (Beal et al., 1993a-c, 1994) and 3-nitropropionic acid (3-NP) (Gould and Gustine, 1982; Hamilton and Gould, 1987) inhibit SDH. Injection of both malonate and 3-NP directly into brain produces damage to neurons and other cells (Ludolf et al., 1991, 1992; Beal et al., 1993a-c, 1994; Fu et al., 1995a,b; Greene and Greenamyre, 1995a,b; Zeevalk et al., 1995). In people who have eaten sugar cane contaminated with 3-NP (Fu et al., 1995a,b; He et al., 1995), taken large doses of 3-NP (He et al., 1995), or have been poisoned with the mitochondrial toxin cyanide (Finelli et al., 1981; Rosenow et al., 1995), damage localized to the basal ganglia occurs that seems to be identical to the brain lesions that occur in patients with MCM mutations (Gebarski et al., 1983; Roodhooft et al., 1990; Gerhardt et al., 1991; Stockler et al., 1992; Brismar and Ozand, 1994; He et al., 1995). In culture, 3-NP acid affects neurons more than glia and can induce neuronal apoptosis (Behrens et al., 1995).

These data suggest that SDH-inhibiting compounds share the ability to kill neurons and that the striatum and globus pallidus seem to be the brain regions most vulnerable to this type of injury. The injury could be attributable to the combined action of Kreb's cycle and mitochondrial electron transport chain inhibition (Fig. 9) as well as a local acidosis produced by the increased flux of 

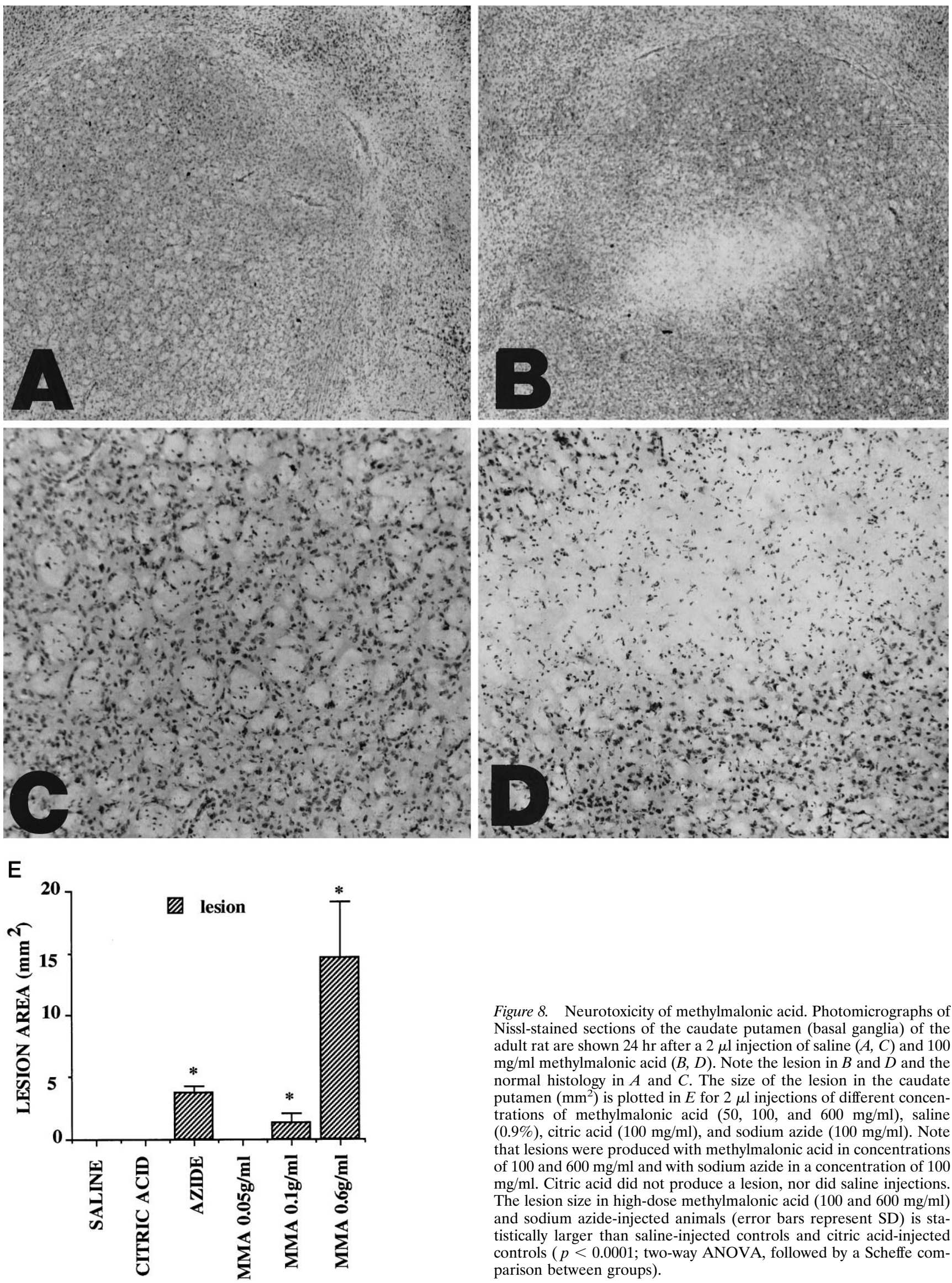

Figure 8. Neurotoxicity of methylmalonic acid. Photomicrographs of Nissl-stained sections of the caudate putamen (basal ganglia) of the adult rat are shown $24 \mathrm{hr}$ after a $2 \mu \mathrm{l}$ injection of saline $(A, C)$ and 100 $\mathrm{mg} / \mathrm{ml}$ methylmalonic acid $(B, D)$. Note the lesion in $B$ and $D$ and the normal histology in $A$ and $C$. The size of the lesion in the caudate putamen $\left(\mathrm{mm}^{2}\right)$ is plotted in $E$ for $2 \mu$ injections of different concentrations of methylmalonic acid $(50,100$, and $600 \mathrm{mg} / \mathrm{ml})$, saline $(0.9 \%)$, citric acid $(100 \mathrm{mg} / \mathrm{ml})$, and sodium azide $(100 \mathrm{mg} / \mathrm{ml})$. Note that lesions were produced with methylmalonic acid in concentrations of 100 and $600 \mathrm{mg} / \mathrm{ml}$ and with sodium azide in a concentration of 100 $\mathrm{mg} / \mathrm{ml}$. Citric acid did not produce a lesion, nor did saline injections. The lesion size in high-dose methylmalonic acid (100 and $600 \mathrm{mg} / \mathrm{ml})$ and sodium azide-injected animals (error bars represent SD) is statistically larger than saline-injected controls and citric acid-injected controls $(p<0.0001$; two-way ANOVA, followed by a Scheffe comparison between groups). 




SUCCINYL-CoA $\longrightarrow$ SUCCINATE

FUMARATE

Figure 9. A diagram showing the relationship among ischemia, branched-chain amino acids, odd-chain fatty acids, and methylmalonyl-CoA mutase metabolism. The inhibitory sites of methylmalonic acid on succinate dehydrogenase $(S D H)$ in the Kreb's cycle and the electron transport chain are also pictured. Methylmalonic acid and malonate are found in brain, inhibit SDH, and can produce brain lesions at high concentrations. 3-Nitropropionic acid (3-NPA) is an exogenous toxin that inhibits SDH and also produces brain lesions. A dashed line is used between ischemia and odd-chain fatty acids to indicate a likely but unproven pathway.

glucose to lactate to increase energy availability. The GABAergic neurons in the striatum may be particularly vulnerable to methylmalonic acid and other SDH inhibitors, because these compounds would decrease availability of the inhibitory amino acid neurotransmitter GABA via blockade of the Kreb's cycle and the GABA shunt (Hassel and Sonnewald, 1995).

Neurological abnormalities occur in all conditions in which methylmalonic acid accumulates, including MCM mutations, inborn errors of cobalamin metabolism, and acquired $\mathrm{B}_{12}$ deficiency (Rosenberg, 1983). Because cobalamin disorders affect mainly white matter, whereas MCM mutations produce severe gray and white matter abnormalities, it has been assumed that these disorders have a different pathogenesis. It is possible, however, that the white matter abnormality observed in $\mathrm{B}_{12}$ deficiency represents the mildest effects of chronic inhibition of SDH and other brain enzymes by methylmalonic acid, whereas the severe brain abnormalities in MCM mutations represent the most severe effects of inhibition of the same enzymes.

\section{REFERENCES}

Allen KL, Almeida A, Bates TE, Clark JB (1995) Changes in respiratory chain activity in mitochondrial and synaptosomal fractions isolated from the gerbil brain after graded ischemia. J Neurochem 64:2222-2229.
Barks JD, Silverstein FS (1992) Excitatory amino acids contribute to the pathogenesis of perinatal hypoxic-ischemic brain injury. Brain Pathol 2:235-243.

Bazan NG (1970) Effects of ischemia and electroconvulsive shock on free fatty acid pool in the brain. Biochim Biophys Acta 218:1-10.

Beal MF, Brouillet E, Jenkins B, Henshaw R, Rosen B, Hyman BT (1993a) Age-dependent striatal excitotoxic lesions produced by the endogenous mitochondrial inhibitor malonate. $\mathrm{J}$ Neurochem 61:1147-1150.

Beal MF, Brouillet E, Jenkins BG, Ferrante RJ, Kowall NW, Miller JM, Storey E, Srivastava R, Rosen BR, Hyman BT (1993b) Neurochemical and histological characterization of striatal excitotoxic lesions produced by the mitochondrial toxin 3-nitropropionic acid. J Neurosci 13:4181-4192.

Beal MF, Hyman BT, Koroshetz W (1993c) Do defects in mitochondrial energy metabolism underlie the pathology of neurodegenerative diseases? Trends Neurosci 16:125-131.

Beal MF, Henshaw DR, Jenkins BG, Rosen BR, Schulz JB (1994) Coenzyme Q10 and nicotinamide block striatal lesions produced by the mitochondrial toxin malonate. Ann Neurol 36:882-888.

Behrens MI, Koh J, Canzoniero LM, Sensi SL, Csernansky CA, Choi DW (1995) 3-Nitropropionic acid induces apoptosis in cultured striatal and cortical neurons. NeuroReport 6:545-548.

Brismar J, Ozand PT (1994) CT and MR of the brain in the diagnosis of organic acidemias. Experiences from 107 patients. Brain Dev $16: 104-124$. 
Brown IR, Rush S, Ivy GO (1989) Induction of a heat-shock gene at the site of tissue injury in the rat brain. Neuron 2:1559-1564.

Crane AM, Jansen R, Andrews ER, Ledley FD (1992) Cloning and expression of a mutant methylmalonyl-coenzyme A mutase with altered cobalamin affinity that causes mut ${ }^{-}$methylmalonic aciduria. J Clin Invest 89:385-391.

Dickson AC, McEvoy JA, Koeppen AH (1994) The cellular localization of malonyl-coenzyme A decarboxylase in rat brain. Neurochem Res 19:1271-1276.

Drejer J, Benveniste H, Diemer NH, Schousboe A (1985) Cellular origin of ischemia-induced glutamate release from brain tissue in vivo and in vitro. J Neurochem 45:145-151.

Dutra JC, Wajner M, Wannmacher CF, Dutra-Filho CS, Wannmacher CMD (1991) Effects of methylmalonate and propionate on uptake of glucose and ketone bodies in vitro by brain of developing rats. Biochem Med Metab Biol 45:56-64.

Dutra JC, Dutra-Filho CS, Cardozo SE, Wannmacher CM, Sarkis JJ, Wajner M (1993) Inhibition of succinate dehydrogenase and betahydroxybutyrate dehydrogenase activities by methylmalonate in brain and liver of developing rats. J Inherit Metab Dis 16:147-153.

Erecinska M, Nelson D, Wilson DF, Silver IA (1984) Neurotransmitter amino acids in the CNS. I. Regional changes in amino acid levels in rat brain during ischemia and reperfusion. Brain Res 304:9-22.

Fenton WA, Hack AM, Helfgott D, Rosenberg LE (1984) Biogenesis of the mitochondrial enzyme methylmalonyl-CoA mutase. Synthesis and processing of a precursor in a cell-free system and in cultured cells. J Biol Chem 259:6616-6621.

Finelli PF (1981) Changes in the basal ganglia following cyanide poisoning. J Comput Assist Tomogr 5:755-756.

Fu Y, He F, Zhang S, Jiao X (1995a) Consistent striatal damage in rats induced by 3-nitropropionic acid and cultures of arthrinium fungus. Neurotoxicol Teratol 17:413-418.

Fu Y, He F, Zhang S, Huang J, Zhang J, Jiao X (1995b) 3-Nitropropionic acid produces indirect excitotoxic damage to rat striatum. Neurotoxicol Teratol 17:333-339.

Gebarski SS, Gabrielsen TO, Knake JE, Latack JT (1983) Cerebral CT findings in methylmalonic and propionic acidemias. Am J Neurol Res 4:955-957.

Gerhardt M, Burke EM, Brandt IK, Crabb DW (1991) Methylmalonic aciduria presenting in an adult. J Inherit Metab Dis 14:113-114.

Gonzalez MF, Shiraishi K, Hisanaga K, Sagar SM, Mandabach M, Sharp FR (1989) Heat-shock proteins as markers of neural injury. Mol Brain Res 6:93-100.

Gould DH, Gustine DL (1982) Basal ganglia degeneration, myelin alteration, and enzyme inhibition induced in mice by the plant toxin 3-nitropropionic acid. Neuropathol Appl Neurobiol 8:377-393.

Greene JG, Greenamyre JT (1995a) Exacerbation of NMDA, AMPA, and L-glutamate excitotoxicity by the succinate dehydrogenase inhibitor malonate. J Neurochem 64:2332-2338.

Greene JG, Greenamyre JT (1995b) Manipulation of membrane potential modulates malonate-induced striatal excitotoxicity in vivo. J Neurochem 66:637-643.

Greene JG, Porter RHP, Eller RV, Greenamyre JT (1993) Inhibition of succinic dehydrogenase by malonic acid produces an "excitotoxic" lesion in rat striatum. J Neurochem 61:1151-1154.

Halperin ML, Schiller CM, Fritz IB (1971) The inhibition by methylmalonic acid of malate transport by the dicarboxylate carrier in rat liver mitochondria. J Clin Invest 50:2276-2282.

Hamilton BF, Gould DH (1987) Nature and distribution of brain lesions in rats intoxicated with 3-nitropropionic acid: a type of hypoxic (energydeficient) brain damage. Acta Neuropathol (Berl) 72:286-297.

Harper AE, Miller RH, Block KP (1984) Branched-chain amino acid metabolism. Annu Rev Nutr 4:409-454.

Hassel B, Sonnewald U (1995) Selective inhibition of the TCA cycle of GABA neurons with 3-nitropropionic acid in vivo. J Neurochem 65:1184-1191.

He F, Zhang S, Qian F, Zhang C (1995) Delayed dystonia with striatal CT leucencies induced by a mycotoxin (3-nitropropionic acid). Neurology 45:2178-2183.

Henshaw T, Jenkins BG, Schulz JB, Ferrante RJ, Kowall NW, Rosen BR, Beal MF (1994) Malonate produces striatal lesions by indirect NMDA receptor activation. Brain Res 647:161-166.

Hertz L (1990) In vitro studies of astrocytes. In: Molecular aspects of development and aging in the nervous system (Lauder JM, Private A, Giacobini E, Vernadakis A, eds), pp 227-243. New York: Plenum.
Hertz L, Juurlink BHJ, Suchet S (1985) Cell cultures. In: Handbook of Neurochemistry, Vol. 8 (Lajtha A, ed), pp 603-661. New York: Plenum. Kinouchi H, Sharp FR, Hill MP, Koistinaho J, Sagar SM, Chan PH (1993) Induction of $72 \mathrm{kDa}$ heat-shock protein and hsp70 mRNA following focal cerebral ischemia in the rat. J Cereb Blood Flow Metab 13:105-115.

Kirino T (1982) Delayed neuronal death in the gerbil hippocampus following ischemia. Brain Res 239:57-69.

Kirino T, Sano K (1984) Selective vulnerability in the gerbil hippocampus following transient ischemia. Acta Neuropathol (Berl) 62:201-208.

Kirschner PB, Henshaw R, Weise J, Trubetskoy V, Finkelstein S, Schulz JB, Beal MF (1995) Basic fibroblast growth factor protects against excitotoxicity and chemical hypoxia in both neonatal and adult rats. J Cereb Blood Flow Metab 15:619-623.

Kishimoto Y, Williams M, Moser HW, Hignite C, Bierman K (1973) Branched-chain and odd-numbered fatty acids and aldehydes in the nervous system of a patient with deranged vitamin $B_{12}$ metabolism. J Lipid Res 14:69-77.

Kolhouse JF, Utley C, Allen RH (1980) Isolation and characterization of methylmalonyl-CoA mutase from human placenta. J Biol Chem 255:2708-2712.

Ledley FD (1990) Perspectives on methylmalonic acidemia resulting from molecular cloning of methylmalonyl-CoA mutase. BioEssays 12:335-340.

Ledley FD, Lumetta M, Nguyen PN, Kolhouse JF, Allen RH (1988) Molecular cloning of L-methylmalonyl-CoA mutase: gene transfer and analysis of mutt cell lines. Proc Natl Acad Sci USA 85:3518-3521.

Ledley FD, Jansen R, Nham SU, Fenton WA, Rosenberg LE (1990) Mutation eliminating mitochondrial leader sequence of methylmalonylCoA mutase causes muto methylmalonic acidemia. Proc Natl Acad Sci USA 87:3147-3150.

Lee WH, Bondy CA (1993) Ischemic injury induces brain glucose transporter gene expression. Endocrinology 133:2540-2544.

Lindquist S (1994) Heat-shock proteins and stress tolerance in microorganisms. Curr Opin Genet Dev 2:748-755.

Lopex-Lahoya J, Garcia ML, Benavides J, Ugarte M (1981) Inhibition by methylmalonate of glycine uptake by synaptosomes from rat spinal cord. J Neurochem 36:325-327.

Ludolf AC, He F, Spencer PS, Hammerstad J, Sabri M (1991) 3-Nitropropionic acid-exogenous animal neurotoxin and possible human striatal toxin. Can J Neurol Sci 18:492-498.

Ludolf AC, Seeling M, Ludolf A (1992) 3-Nitropropionic acid decreases cellular energy levels and causes neuronal degeneration in cortical explants. Neurodegeneration 1:155-161.

Maniatis R, Fritsch EG, Sambrook J 1987 Molecular cloning: a laboratory manual. Cold Spring Harbor, NY: Cold Spring Harbor Laboratory.

Massa SM, Longo FM, Zuo J, Wang S, Chen J, Sharp FR (1995) Cloning of rat grp75, an hsp70-family member, and its expression in normal and ischemic brain. J Neurosci Res 40:807-819.

Massa SM, Swanson RA, Sharp FR (1996) The stress gene response in brain. Cereb Brain Metab Rev 8:95-158.

Matsui SM, Mahoney MJ, Rosenberg LE (1983) The natural history of the inherited methylmalonic acidemias. N Engl J Med 308:857-861.

Mitzen EJ, Koeppen AH (1984) Malonate, malonyl-coenzyme A, and acetyl-coenzyme A in developing rat brain. J Neurochem 43:499-506.

Nakano S, Kogure K, Abe K, Abe T (1990) Ischemia-induced alterations in lipid metabolism of the gerbil cerebral cortex. I. Changes in free fatty acid liberation. J Neurochem 54:1911-1916.

Nowak Jr TS, Bond U, Schlesinger MJ (1990) Heat-shock RNA levels in brain and other tissues after hyperthermia and transient ischemia. J Neurochem 54:451-458.

Ostergaard JR, Reske-Nielsen E, Nathan E, Rasmussen K (1991) Incomplete development of the brain in a newborn with methylmalonic aciduria. Clin Neuropathol 10:85-92.

Riepe M, Hori N, Ludolf AC, Carpenter DO, Spencer PS, Allen CN (1992) Inhibition of energy metabolism by 3-nitropropionic acid activates ATP-sensitive potassium channels. Brain Res 586:61-66.

Riley KM, Dickson AC, Koeppen AH (1991) The origin of free brain malonate. Neurochem Res 16:117-122.

Roodhooft AM, Baumgartner ER, Martin JJ, Blom W, Van Acker KJ (1990) Symmetrical necrosis of the basal ganglia in methylmalonic acidemia. Eur J Pediatr 149:582-584.

Rosenberg LE (1983) Disorders of propionate and methylmalonate metabolism. In: The metabolic basis of inherited disease, 5th Ed (Stanbury 
JB, Wyngaarden JB, Fredrickson DS, Goldstein JL, Brown MS, eds), pp 474-497. New York: McGraw-Hill.

Rosenow F, Herholz K, Lanfermann H, Weuthen G, Ebner R, Kessler J, Ghaemi M, Heiss W-D (1995) Neurological sequelae of cyanide intoxication - the patterns of clinical, magnetic resonance imaging, and positron emission tomography findings. Ann Neurol 38:825-828.

Sbai D, Narcy C, Thompson GN, Mariotti A, Saudubray JM, Bresson JL (1994) Contribution of odd-chain fatty acid oxidation to propionate production in disorders of propionate metabolism. Am J Clin Nutr 59:1332-1337.

Stockler S, Slavc I, Ebner F, Baumbartner R (1992) Asymptomatic lesions of the basal ganglia in a patient with methylmalonic aciduria. Eur J Pediatr 151:920-921.

Sum JM, Twiss JL, Horoupian DS (1993) Selective death of immature neurons in methylmalonic acidemia of the neonate: a case report. Acta Neuropathol (Berl) 85:217-221.

Swanson RA, Sharp FR (1992) Zinc induction of HSP72 expression and cell death in primary astrocyte culture. Glia 6:198-205.

Toyashima S, Watanabe F, Saido H, Miyatake H, Nakano Y (1995) Methylmalonic acid inhibits respiration in rat liver mitochondria. J Nutr 125:2846-2850.

Vannucci SJ, Seaman LB, Vannucci RC (1996) Effects of hypoxiaischemia on GLUT1 and GLUT3 glucose transporters in immature rat brain. J Cereb Blood Flow Metab 16:77-81.
Wajner M, Dutra JC, Cardozo SE, Wannmacher CM, Motta ER (1992) Effect of methylmalonate on in vitro lactate release and carbon dioxide production by brain of suckling rats. J Inherit Metab Dis 15:92-96.

Wendel U, Zass R, Leupold D (1993) Contribution of odd-numbered fatty acid oxidation to propionate production in neonates with methylmalonic and propionic acidemias. Eur J Pediatr 152:1021-1023.

Wilkemeyer MF, Crane AM, Ledley FD (1990) Primary structure and activity of mouse methylmalonyl-CoA mutase. Biochem $\mathrm{J}$ 271:449-455,

Wilkemeyer MF, Andrews ER, Ledley FD (1993) Genomic structure of murine methylmalonyl-CoA mutase: evidence for genetic and epigenetic mechanisms determining enzyme activity. Biochem J 296:663-670.

Wullner U, Young AB, Penney JB, Beal MF (1994) 3-Nitropropionic acid toxicity in the striatum. J Neurochem 63:1772-1781.

Yoshida S, Ikeda N, Busto P, Santiso M, Martinez E, Ginsberg MD (1986) Cerebral phosphoinositide, triacylglycerol, and energy metabolism in reversible ischemia: origin and fate of free fatty acids. J Neurochem 47:744-757.

Zeevalk GD, Derr-Yellin E, Nicklas WJ (1995) Relative vulnerability of dopamine and GABA neurons in mesencephalic culture to inhibition of succinate dehydrogenase by malonate and 3-nitropropionic acid and protection by NMDA receptor blockade. J Pharmacol Exp Ther 275: $1124-1130$ 